\title{
Phaeochromocytoma with nocturnal elevation of blood pressure
}

\author{
TOSHIHIDE YAMAMOTO \\ M.D.
}

\author{
TADAFUMI Jo \\ M.D.
}

\author{
TOSHIMOTO ISHIBASHI \\ M.D. \\ Center for Adult Diseases, Osaka
}

\begin{abstract}
Summary
A 47-year-old woman with phaeochromocytoma showed blood pressure changes characterized by the regular appearance of hypertension at night. The urinary excretion of catecholamines and their metabolites showed augmentation roughly parallel with the elevation of the blood pressure. During normotensive periods, the urinary excretion of catecholamines and their metabolites was elevated. The findings suggested that the pressor effect of catecholamine hypersecretion was compromised to a certain degree and that the regular nocturnal appearances of hypertensive paroxysm conceivably resulted from a spontaneous cyclic augmentation of the catecholamine secretion from the tumour.
\end{abstract}

\section{Introduction}

Phaeochromocytoma is a tumour arising from the chromaffin tissue which secretes catecholamines. The hypertension of this disorder is either paroxysmal or sustained, and has been generally thought to be resulting from either episodic or continuous secretion of catecholamines from the tumour (Crout, Pisano and Sjoerdsma, 1961; Gifford et al., 1964; Sheps et al., 1966). In certain cases, however, increased secretion of them was not accompanied by hypertensive episodes (Sato et al., 1971; Taubman, Pearson and Anton, 1974). In the following communication, a patient with phaeochromocytoma with nightly occurrence of hypertension is described, in whom the relation between hypertension and catecholamine secretion was sought by measurements of urinary catecholamines and their metabolites in timed urine samples and of the blood pressure every two hours for 2 consecutive days before and after the removal of the tumour.

\section{Case report}

A 47-year-old housewife was referred to the authors for evaluation of hypertension of about 3

Correspondence: Dr Toshihide Yamamoto, Kishiwada Tokushukai Hospital, 330 Isonakami-cho, Kishiwada, Osaka, Japan (596). years' duration. Her hypertension was characterizedo by a marked variation of its level, the blood pressure being high in the morning and almost normal in the afternoon. She had had frequent headache, worse on awakening and in the early morning. She had excessive perspiration and frequent post-prandiato epigastric fullness as well. She had been admitted to응 hospital elsewhere for a gastric ulcer 4 monthspreviously. There was no family history of phaeo-c chromocytoma or related disorders.

The patient was a well developed, well nourished woman, who weighed $62 \mathrm{~kg}$ and was $159 \mathrm{~cm}$ in height. Her blood pressure on one occasion wags $170 / 120 \mathrm{mmHg}$ in the supine position and $122 \% 0^{\circ 0}$ $\mathrm{mmHg}$ in the upright position. A few small haemo rhages as well as moderate degree of arteriolaris spasm were observed in the fundi. The skin waso warm and moderate perspiration was noted over theo anterior chest. The rest of the physical examination was unremarkable.

Urinalysis showed ++ proteinuria and $\pm \frac{\overline{0}}{3}$ glycosuria. Haemoglobin was $15.7 \mathrm{~g} / \mathrm{dl}$ and haematocrit was $47 \cdot 2 \%$. The chest X-ray revealedo left ventricular enlargement and an ECG showed lef ventricular hypertrophy. Urinary excretion of free catecholamines, free and conjugated (total) meta-onephrine and normetanephrine (hence, total meta nephrines, tMN) and vanilmandelic acid (VMA) foro 3 consecutive days were as follows: free adrenaline, $0,80,20 \mu \mathrm{g} /$ day; free noradrenaline, 2325, 2095, $2632 \mu \mathrm{g} /$ day; tMN, 4600, 5000, $3700 \mu \mathrm{g} /$ day; VMA $14.8,20.0,14.6 \mathrm{mg} /$ day; phentolamine mesylate elicited hypotensive reaction when injected i.v. in the hypertensive phase. The i.v. injection of glucagon ${ }^{\circ}$ caused a significant elevation of blood pressures during the hypertensive phase but the same dose caused a lesser degree of hypertensive response wheng given in the normotensive phase (Fig. 1). Theo abdominal angiography disclosed a vascular massर in the vicinity of the right adrenal gland. The ora? cholecystogram failed to visualize the gall bladdero but an ultrasonic echogram of it suggested the pres-웅 ence of multiple small grains. 


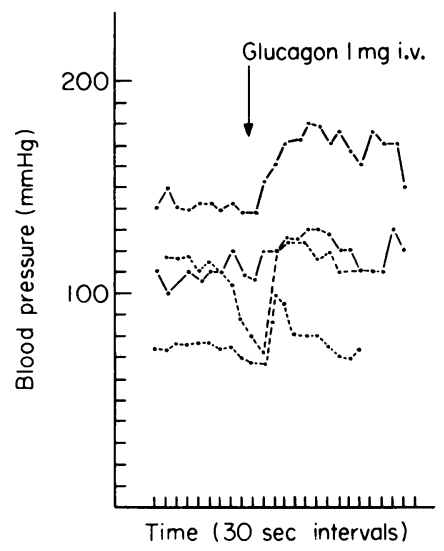

Fig. 1. Blood pressure response to glucagon in hypertensive and normotensive phases. -0 , in hypertensive phase.

Her blood pressure (BP) varied widely as seen in Fig. 2, and had a daily peak from late in the evening until the following morning. She was relatively free from symptoms but she complained of headache and perspiration in the morning. On laparotomy a mass was found in the right adrenal gland, partly situated deep behind the inferior vena cava, which was excised. The removed mass was round and $6 \mathrm{~cm}$ in diameter, $38 \mathrm{~g}$ in weight. The histology of the tumour was consistent with that of phaeochromocytoma. Postoperatively, BP was stabilized at the normal level. The patient, however, was troubled by persisting nausea and vomiting of variable severity, recurrence of the gastric ulcer and at least one episode of acute cholecystitis, which necessitated subtotal gastrectomy with Bilroth II anastomosis and cholecystectomy. She recovered from the second laparotomy and was discharged.

\section{Special studies}

Because of the marked variation of BP during $24 \mathrm{hr}$, the relation between BP and urinary excretion of catecholamines and their metabolites were studied before and after the removal of the tumour.

\section{Materials and methods}

BP was measured in the supine position using a sphygmomanometer every $2 \mathrm{hr}$ for two consecutive days before and after the resection of the phaeochromocytoma. On the days when BP was measured every 2 hours, urine was collected every 4 hours in a jar containing hydrochloric acid. The aliquots of 4-hr urine specimens were stored at $-20^{\circ} \mathrm{C}$ until the time of analysis. To make urine collection exactly every 4 hours, the patient was instructed by a nurse to void at the end of each 4-hr period. Urinary free catecholamines (fCA) were measured by trihydroxyindol method using a commercial alumina column according to the manufacturer's instruction the results of which were expressed as $\mu \mathrm{g}$ of noradrenaline. tMN was measured by the method of Pisano (1960) and VMA measured by the method of Pisano Crout and Abraham (1962).

\section{Results}

Before the removal of phaeochromocytoma there was a marked variation of BP, high in the late evening and until the following morning, which was no longer observed after the resection of the tumour. Urinary excretion of catecholamines and their metabolites was elevated all day pre-operatively and was further augmented nightly; this paralleled with paroxysms of hypertension (Fig. 3). Postoperatively the urinary excretion of catecholamines was reduced to the normal level and no apparent diurnal variation was noted.

\section{Discussion}

The hypertension of phaeochromocytoma has been considered to be due to the excessive secretion of catecholamines from the tumour. However, the exact relation of hypertension to catecholamine hypersecretion remained to be determined. The urinary excretion of catecholamines (in certain

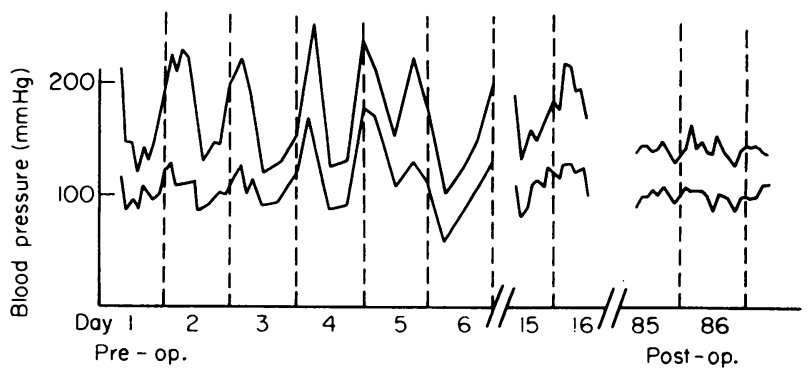

Fig. 2 Profiles of blood pressure before and after removal of phaeochromocytoma. Broken lines indicate midnight. 


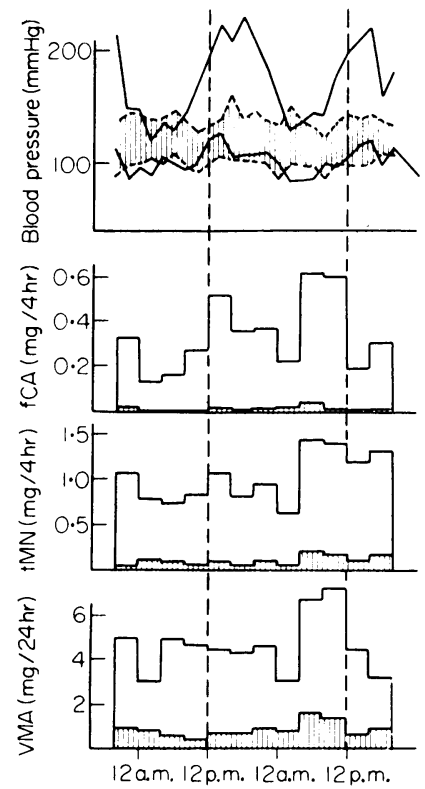

Fig. 3. Profiles of blood pressure and urinary excretion of catecholamines and their metabolites before and after removal of phaeochromocytoma. Shaded areas indicate postoperative results; BP, blood pressure; fCA, free catecholamines; tMN, total metanephrines; VMA, vanilmandelic acid. Broken lines indicate midnight.

cases, metanephrines also) was known to increase following pressor responses to histamine in subjects with phaeochromocytoma (Gifford et al., 1964). Nevertheless, Sato et al. (1971) found elevated urinary excretion of catecholamines in five patients with surgically proved phaeochromocytoma during the pre-operative normotensive periods. Taubman et al. (1974) reported a case of phaeochromocytoma without hypertension despite numerous measurements of BP, whose diagnosis was confirmed by elevated plasma as well as urinary levels of catecholamines and by surgery. In the present case the augmented urinary excretion of catecholamines during the paroxysms of hypertension could be considered as reflecting augmented secretion of noradrenaline from the tumour although the actual plasma level was not measured. Elevated urinary excretion of catecholamines during the normotensive periods suggests that the patient acquired a certain tolerance to the pressor effect of catecholamines. Reduced blood volume in certain patients with this condition (Sjoerdsma, Engelman and Waldman, 1966; Tarazi, Dustan and Frohlich, 1970) or reduced pressor response to circulating noradrenaline (Torikai, 1969) might be operative in preventing the development of hypertension of phaeochromo cytoma to a limited extent. Excessive secretion of catecholamines, however, could not be comprisedand the paroxysm of hypertension might ensue.

A variety of physiological changes was reported as being associated with the initiation of the paroxysms of hypertension in subjects with phaeo $\overline{\bar{n}}$. chromocytoma (e.g. twisting of the trunk, bendingo forward, assuming a decubitus position) as cited bo Hermann and Mornex (1964). None of these appeared responsible for the paroxysms in thẹ present case because of the regularity of the hyper tensive episodes. The nocturnal elevation of BP and supposedly of catecholamine secretion would rather be due to a spontaneous cyclicity of the catechof 3 amine secretion from the tumour.

Finally, the authors stress the importance of frequent measurements of BP, even throughout the night, to detect phaeochromocytoma with paroxys mal hypertension.

\section{Acknowledgment}

We with to express our thanks to Dr Tadao Itoh foe differential measurements of catecholamines of urine samples for three consecutive days. We also thank Miss Kei Doi fof technical assistance and the nursing staffs for the care of the patient.

\section{References}

Crout, J.R., Pisano, J.J. \& Sjoerdsma, A. (1961) Urinarie excretion of catecholamines and their metabolites if pheochromocytoma. American Heart Journal, 61, 375.

Gifford, R.W., Kvale, W.F., Maher, F.T., Roth, G.M. \& Priestley, J.M. (1964) Clinical features, diagnosis an treatment of pheochromocytoma: A review of 76 cases Proceedings. Mayo Clinic, 39, 281.

Herman,, H. \& Mornex, R. (1964) Human Tumouß Secreting Catecholamines. p. 3. Pergamon Press, London. Pisano, J.J. (1960) A simple analysis for normetanephrin and metanephrine in urine. Clinica chimica acta, 5, 406.

Pisano, J.J., Crout, J.R. \& Abraham, D. (1972) Detere mination of 3-methoxy-4-hydroxymandelic acid in urine? Clinica chimica acta, 7, 285.

Sato, T., Ono, I., Miura, Y. \& Yoshinaga, K. (1971ß Increased catecholamine excretion during normotensive phase in paroxysmal type of pheochromocytoma. Japanes? Heart Journal, 12, 214.

Sheps, S.G., Tyce, G.M., Flock, E.V. \& Maher, F.T. (1966q Current experience in the diagnosis of pheochromocytoma Circulation, 34, 473.

Suofrdsma, A., Engelman, K. \& Waldman, T.A. (1966 Pheochromocytoma. Annals of Internal Medicine, 65, 1302 N

Tarazi, R.C., Dustan, H.P. \& Frohlich, E.D. (1970) Plasma volume and chronic hypertension. Archives of Internal Medicine, 125, 835.

Taubman, I., Pearson, O.H. \& Anton, A.H. (1974) Asympw tomatic catecholamine-secreting pheochromocytoma웅 American Journal of Medicine, 57, 953.

TORIKAI, T. (1969) [Aspects of hormones and hypertension. Japanese Journal of Society of Internal Medicine, 58, 578 [in Japanese]. 